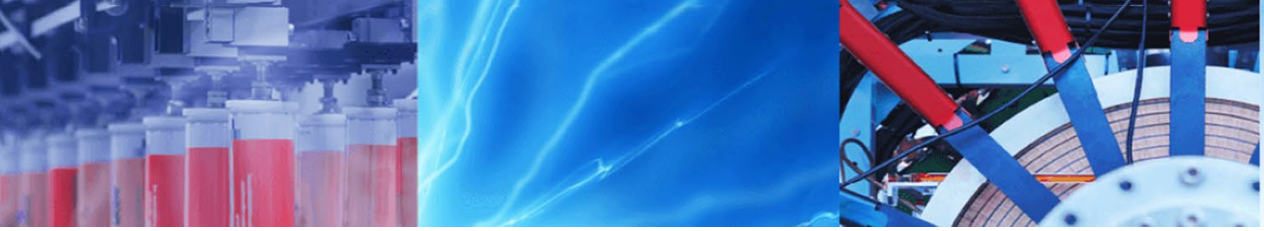

Research Article

\title{
An implementation of error localization algorithms for model calibration of dynamical systems
}

\author{
L. G. Horta ${ }^{1}$ - M. C. Reaves ${ }^{1}$
}

Received: 3 December 2019 / Accepted: 11 April 2020 / Published online: 6 May 2020

(c) This is a U.S. Government work and not under copyright protection in the U.S.; foreign copyright protection may apply 2020

\begin{abstract}
The paper discusses the implementation of three Error Localization Algorithms (ELA), test assessment metrics, and an example of model calibration. During model calibration, users routinely perform sensitivity studies to determine critical parameters for model adjustments. Although this makes engineering sense, sensitivity information only reflects parameter importance and not necessarily model deficiencies. Instead, the task of identifying model deficiencies is best suited for ELA in which measured data is used directly to identify potential problem areas in the model. Three ELA methods are applied in this work: (1) Coordinate Modal Assurance Criterion (CoMAC), (2) Robust Model Error, and (3) Analytical Dynamic Model Improvement (ADMI). To establish credibility of the ELA approaches, data from two analytical examples are used with ELA to identify known problem areas. Of the three ELA approaches implemented, only ADMI correctly identified known problem areas in the model. An example application of the ADMI algorithm is presented to identify problem areas of an isogrid-panel model from experimental data. These identified areas are then used to calibrate a model.
\end{abstract}

Keywords Error localization $\cdot$ Modal test $\cdot$ Model calibration

\section{Introduction}

Model calibration of dynamical systems is a very important activity undertaken for the verification and validation of dynamic models. Calibrated models are used for flight loads assessments and stability analysis. The importance of this task has prompted numerous excellent papers and books on the subject (see for example [1] and [2]), including the development of commercial programs to automate the calibration process. Unfortunately, at times users have unrealistic expectations about the process and their ability to correct model deficiencies using existing tools.

Computer advances in the last 30 years and the development of finite element analysis (FEA) allow engineers to create detailed high fidelity models to predict the dynamic behavior of very complex systems. During the development of such models, contributions from many different areas are brought together in terms of material/ mechanical properties, modeling techniques, and different modeling approaches, to develop Finite Element Models (FEM), which are later validated through carefully crafted tests. Because contributions from many engineers are often integrated into the model at various stages of the hardware development, the task of model calibration, as defined in $[3,4]$, often expect model developers to come together to correct model deficiencies. Although this seems like a reasonable expectation, what is more likely to happen is that the model calibration task is handed-over to an analyst, knowledgeable about the commercial FEA code, but not necessarily familiar with the model development. At a disadvantage from the onset, the analyst first step is to evaluate the adequacy of the model in terms of established metrics. For dynamic problems, goodness metrics are normally expressed in terms of frequency errors between test and analysis (see [5]), cross orthogonality errors (see $[6,7])$, and at times frequency response

L. G. Horta, lucas.g.horta@nasa.gov | 1 NASA Langley Research Center, MS230, Hampton, VA 23681, USA. 
principal values, (see [8]). With automated algorithms to search for parameters to reconcile the model with test, users must rely on engineering judgment, test metrics, sensitivity analysis, and energy analysis to decide what areas of the model are most likely in error. Although these approaches are very powerful, they do not use the test data directly when deciding what areas of the model need to be corrected. To contrast Error Localization Algorithms (ELA), which use test data directly, have been developed for that purpose.

Perhaps one of the earliest ELA is the Coordinate Modal Assurance Criterion (CoMAC) [5]. CoMAC was developed to study the spatial dependency of the measured degrees of freedom. Specifically, CoMAC looks at the contribution of an individual degree-of-freedom (DOF) to the correlation expression of a particular mode (see [9] and [10]). When used with test and analysis modes, CoMAC provides for a figure of merit to quantify the degree-of-correlation between a measured DOF and the model. Admittedly, lack of correlation can be a reflection of a bad test, but for cases where test practices are adequate, low CoMAC values can point to problem areas in the model. CoMAC is one of three ELA approaches evaluated in this work.

The second ELA approach used here is the Robust Model Error (RME) localization [11]. An outgrowth from the Sensitivity-Based Element-by-Element model update theory [12], RME uses the modal force error vector to determine areas (or zones) in the model exhibiting the largest modal force errors. With zones identified, a subset of the FEM parameters are selected for updating. This approach is the only one that accounts for test uncertainties explicitly.

A third approach to conduct ELA assessment, based work in [13], is referred to as the Analytical Dynamic Model Improvement (ADMI) algorithm. ADMI determines corrections to the nominal model to match measured modes and frequencies. Because the formulation uses a closedform expression to compute model corrections to match the test data, it explicitly determines needed changes to the nominal model. As with many numerical techniques for model corrections, results are heavily dependent on the number of measurements, the number of modes, and the quality of the measured data. However, under ideal conditions ADMI can compute exactly both mass and stiffness corrections needed to reconcile model with test. The novelty and innovation of our work is in recognizing that ADMI effectively solves an inverse problem; that is given test data ADMI provides changes in mass and stiffness to correct the model. Admittedly, ADMI suffers from spill-over problems but by connecting it to the work in [17] the problem is mitigated.

To gain confidence in the ability of ELA methods to identify problem areas in the model, all three approaches have been applied to different models, starting with simple lump-mass-models to more complex FEA models developed using MSC Nastran [14]. For brevity, only two example problems are shown here: (1) a cantilever tube, and (2) an isogrid panel. Models for both cases have been perturbed to assess the ability of ELA to identify correctly problem areas in the model.

The paper presents first a brief description of all three ELA formulations, followed by a description of the test cases used in this study. In order to create simulated experimental data representative of current test practices, optimal sensor placement tools are used to ensure that modes meet orthogonality metrics. With sensors properly placed, pre-test analysis metrics are discussed for each test case along with target modes selections. ELA is first applied to simulated experiments with known problem areas to demonstrate the validity of the approach. Finally, a demonstration of the calibration process using experimental data for an isogrid is discussed.

\section{Error localization algorithms}

\subsection{Coordinate modal assurance criterion (CoMAC)}

CoMAC is the first of three metrics implemented for error localization studies. As the name already suggest, this metric is effectively a variation of the Modal Assurance Criterion, initially proposed in [9], to quantify the correlation level between measured and predicted mode shapes. For example, given a measured shape $\phi$ and an analysis shape $\psi$ for $L$ measured modes, CoMAC for the ith DOF is computed as

$\operatorname{CoMAC}(i)=\frac{\sum_{j=1}^{L}\left(\phi_{j}\left(x_{i}\right) \psi_{j}\left(x_{i}\right)\right)^{2}}{\left(\sum_{j=1}^{L} \phi_{j}\left(x_{i}\right)^{2} \sum_{j=1}^{L} \psi_{j}\left(x_{i}\right)^{2}\right)}$

In this initial formulation, COMAC results are only available at each measured $x_{i}$ DOF. However, using mode shape expansion techniques [15] and [16], users can extrapolate results to the entire model. Although this metric was not developed for the purpose of error localization, lack of correlation at a certain DOF can be interpreted as model error.

\subsection{Robust model error (RME)}

RME localization, the second ELA considered here, is an approach based on a modal force error vector, defined as an un-damped impedance of a nominal FEM at each frequency. Readers are referred to [11] for details on the formulation but the index itself can be easily computed using data from a measured mode $\phi_{j}$ at location $x_{j}$ and the 
corresponding frequency $\omega_{j}$. The modal force error vector is computed as

$R_{i}\left(x_{j}\right)=\left(K-\omega_{i}^{2} M\right)\left\{\begin{array}{l}\phi_{i}^{m}\left(x_{j}\right) \\ \phi_{i}^{o}\left(x_{j}\right)\end{array}\right\}$

or

$-R_{i}\left(x_{j}\right)=\left(\Delta K-\omega_{i}^{2} \Delta M\right)\left\{\begin{array}{l}\phi_{i}^{m}\left(x_{j}\right) \\ \phi_{i}^{o}\left(x_{j}\right)\end{array}\right\}$

where the modal force error is produced from unknown mass and stiffness changes $[\Delta K, \Delta M]$ needed to balance the equation of motion exactly. In this formulation, mode shapes are partitioned into measured (superscript $m$ ) and omitted (superscript o) DOF. The RME formulation normalizes the residual vector index by incorporating measurement errors. Effectively, the index is normalized using the estimated variance as $\widehat{R}_{i}\left(x_{j}\right)=R_{i}\left(x_{j}\right) / \sigma\left(R_{i}\left(x_{j}\right)\right)$ (see [11] for details). With measured modes and frequencies at hand, one can now compute the RME index at each measured DOF. For cases with incomplete data sets, [11] proposed an expansion approach that minimizes the magnitude of the impedance residual $\left\|R_{i}\right\|$. In our implementation, a Guyan expansion is used instead. Note that RME do not compute explicitly the changes in $\Delta K$ and $\Delta M$ required to correct the model.

\subsection{Analytical dynamics model improvement (ADMI)}

ADMI, the third ELA approach implemented here, is derived from the work in [13]. In this approach mass and stiffness corrections are computed from measured modes and frequencies to match the test data exactly. ADMI solves a closed-form quadratic optimization problem to determine corrections to the nominal mass and stiffness matrices that satisfy the symmetry and orthogonality conditions for dynamic problems. In other words, given a set of measured mode shapes $\phi$ and eigenvalues $\Omega$, a solution can be obtained for the changes in $\Delta K$ and $\Delta M$ such that

$((K+\Delta K)-\Omega(M+\Delta M)) \phi=0$

is satisfied exactly. Readers are again referred to [13] for a detail derivation. As with RME and CoMAC, unmeasured (or omitted) DOF are computed using a Guyan expansion. When recovering mass and stiffness corrections, oftentimes model corrections can produce changes to parts of the model not in the measurement set. This problem is referred to as "spill-over" in [17]. The problem occurs because of the use of an incomplete set of modes and frequencies to determine model corrections. Intuitively, the approach needs to correct the model to match the measured modes and frequencies while maintaining other parts of the model unchanged. Reference [17] showed that in order to maintain the positive definiteness properties of $M+\Delta M$ and $K+\Delta K$, the measured eigenvector set needs to be a linear combination of the eigenvectors of the nominal system $[M, K]$. Hence, the measured mode shapes must be spanned by the nominal model mode shapes. The ADMI approach is the only one that provides not only areas of improvements but also the numerical values needed to match the test data.

Because $\Delta K$ and $\Delta M$ are the changes needed to correct the nominal model, users can determine model improvement areas directly from these changes. In fact, in our implementation, changes in mass and stiffness are sorted and ranked in descending order of magnitudes to decide what areas of the model to correct first. In spite of this, realizing those changes through parameters in the FEM is not trivial. To verify the initial implementation, ADMI was used with simple lump-mass models to recover exact changes made to the model mass and stiffness, using simulated test data. Intuitively, the process is straightforward but there are many parameters left to the discretion of the user. Parameters crucial to the ELA success include the number of sensors, sensor locations, input locations, number of modes and frequencies, and model order. In the following, two analytical examples are shown to highlight differences in the three ELA solutions.

\section{Analytical example problems}

A description of the two test cases used in the paper is provided next. The first test-case is a cantilever tube, whereas the second test-case involves two modeling approaches for an isogrid panel. Isogrid panel topology [18] is a construction architecture often used in launch vehicle tanks. Because of the intricate design features of isogrid panels, model developers often use simplified plate-elements with equivalent material properties in lieu of a more detailed FEM. For this reason, models for both equivalent and detailed isogrid panel are considered here.

\subsection{Tube model}

Our first test-case, shown in Fig. 1, is a cantilever tube 36 inches long with a diameter of 2 inches; modeled using the material properties for aluminum. Developed using MSC Nastran, the FEM has 1589 grid points and 768 CQUAD elements. Table 1 list the first eight frequencies of the nominal system with the tube clamped at the bottom. Figure 2 shows shapes for modes 3 and 5 at $355 \mathrm{~Hz}$ and $524 \mathrm{~Hz}$, respectively. 


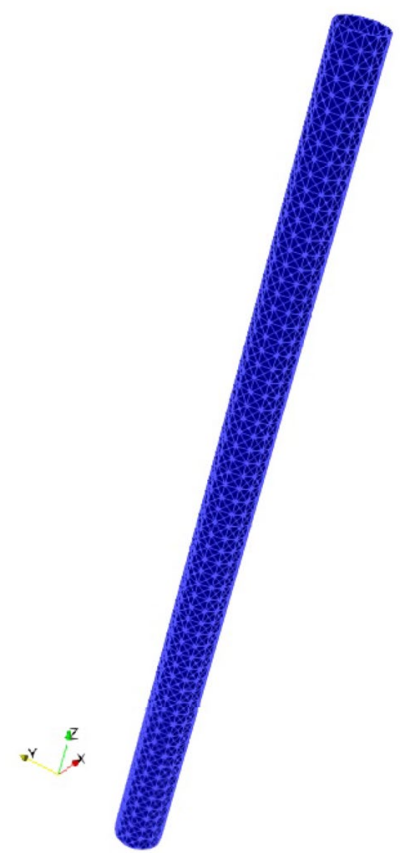

Fig. 1 Tube model

Because ELA depends on accurate mode shapes and frequencies, adequacy of test data is critical. Specifically, measured mode shapes must be distinguishable from one another. For this reason, optimal sensor placement for the tube is conducted and 99 sensors are placed as shown in Fig. 3. This optimal placement minimizes the off-diagonal values in the self-orthogonality (SO) matrix (see [6] for definitions), to make modes distinguishable. Figure 4 shows the rectangular SO results using 99 sensors; the ordinate shows 8 target modes and the abscissa shows the first 20 modes in the model. Optimal placement produced SO off-diagonal values less than $5 \%$ for all target modes. A rectangular SO metric is used to ensure that modes are distinguishable over a much broader range of frequencies.

To evaluate ELA, the tube model is perturbed in the red region shown in Fig. 5. Model perturbations caused modes and frequencies to shift, as shown in Table 1 under perturbed frequencies. Mode shape changes are best compared using Cross-Orthogonality (CO) between modes from the perturbed and nominal model. Figure 6 shows $\mathrm{CO}$ results; perturbed along the ordinate and nominal across the abscissa. Because off-diagonal terms are not zero, it is apparent that the perturbed shapes have significantly changed.

\subsection{Discussion of results with tube model}

Results showing the identified problem areas from applying all three ELA approaches using 10 modes and 99 sensors, are shown in Fig. 7. From left to right are CoMAC, RME, and ADMI results, respectively. Two comments are in order: (1) Readers are cautioned that the numerical values from

Fig. 2 Analysis modes 3 and 5
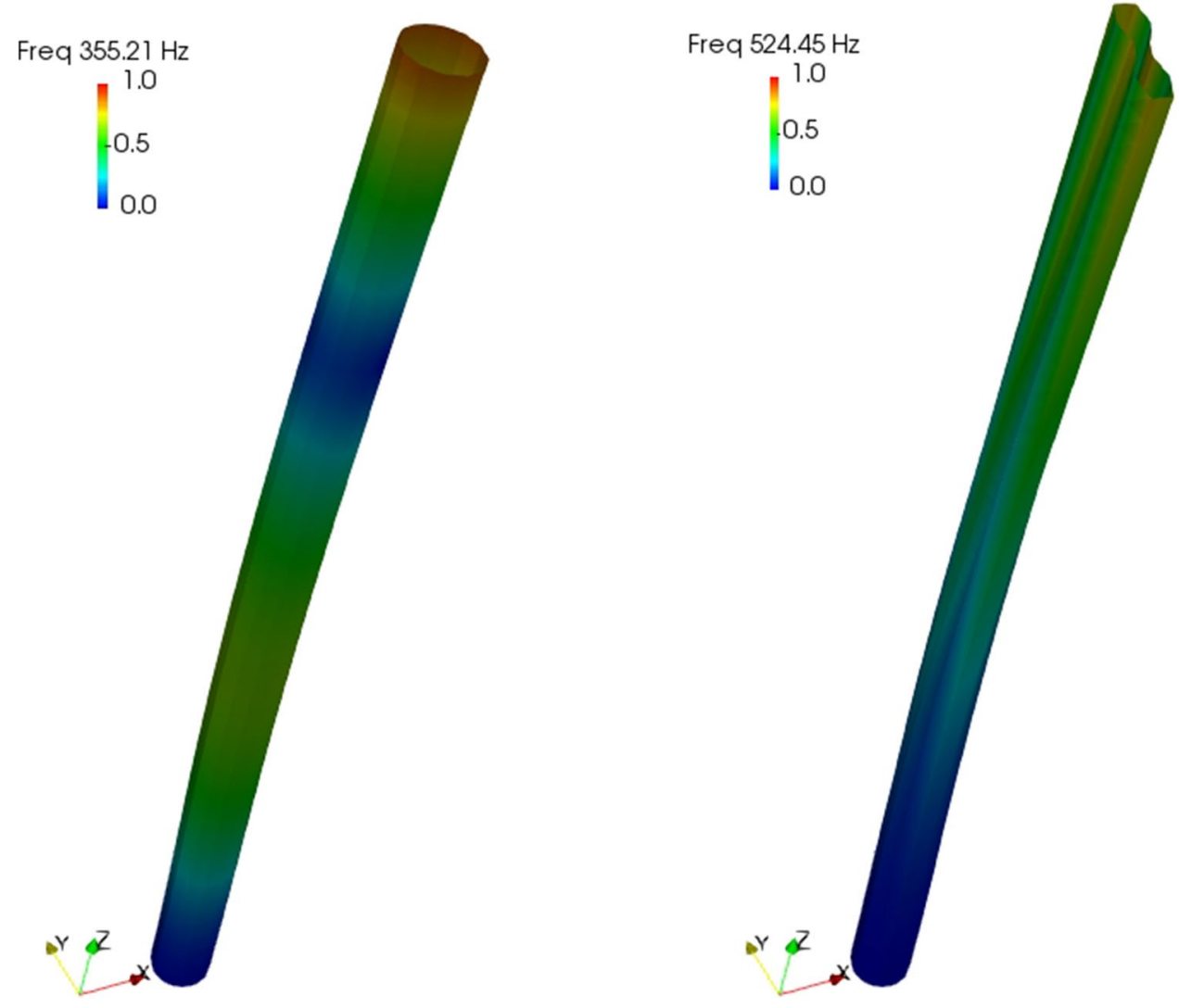
Table 1 Tube modal frequencies

\begin{tabular}{lcc}
\hline Mode No. & Nominal Freq. $(\mathrm{Hz})$ & $\begin{array}{l}\text { Perturbed } \\
\text { Freq. }(\mathrm{Hz})\end{array}$ \\
\hline 1 & 58.4 & 50.2 \\
2 & 58.4 & 51.7 \\
3 & 355.2 & 353.7 \\
4 & 355.2 & 355.1 \\
5 & 524.4 & 523.9 \\
6 & 524.7 & 524.1 \\
7 & 538.4 & 533.2 \\
8 & 545.3 & 534.7 \\
\hline
\end{tabular}

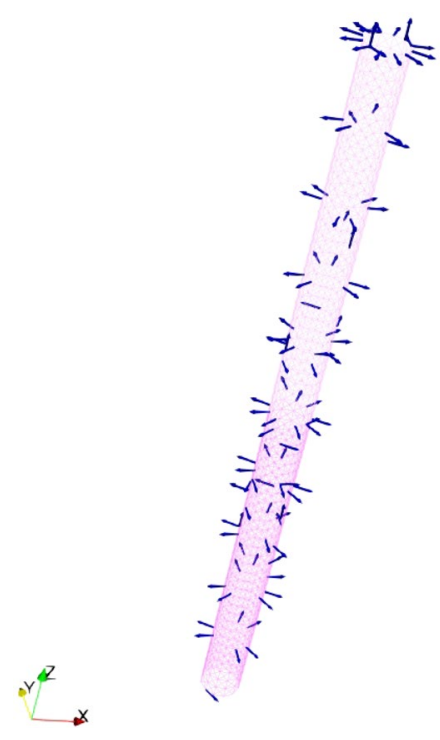

Fig. 3 Optimal sensor placement with 99 sensors

each method correspond to completely different metrics, however, their magnitude should point to problem areas of the model, and (2) to facilitate viewing of problem areas, ELA results are displayed using the elements of the FEM (e.g., CQUAD4) and are colored according to magnitudes. Comparing these results to the known perturbation shown in Fig. 5, note that the ADMI algorithm is the only method that not only identifies correctly the area where model changes occurred, but is also called for stiffness reductions in those areas. Moreover, $\mathrm{CO}$ results using the corrected mass and stiffness computed using ADMI, not shown here, matched exactly the shapes and frequencies from the perturbed model. Results here use a nominal model with 2340 DOF, hence, perturbed mode shapes are expanded using a Guyan expansion from 99 to 2340 . Attempts to implement ELA without expanding the shapes failed. In addition, when the nominal model grid is coarse, identifying problem areas in the model is practically impossible. Although the ability to correct the model is not guaranteed, this process provides users with a starting point based on measured data. When users rely on sensitivity and energy analysis to select improvement areas, it is very likely that the correct problem areas will not be identified. For instance, the application of standard sensitivity analysis on this problem is likely to point to the support boundary as a problem area.

\subsection{Rectangular isogrid panel}

The second test-case discussed here is motivated by a study conducted at NASA Langley to evaluate the adequacy of using equivalent panel properties, derived from static approximations, to model the dynamic behavior of isogrid panels. Figure 8 shows a picture of a rectangular isogrid panel with dimensions $8 \times 141 / 8$ inches, $3 / 32$ inches nominal panel skin thickness, and a total weight of $1.35 \mathrm{lbf}$. For small panels, modeling the complex pattern of the ribs is not difficult but for large panels, the computational complexity is often large. In 1973, the McDonald Douglas Astronautics Company under contract for NASA [18] developed a computational handbook with formulas to estimate the equivalent properties for isogrid designs to approximate their static behavior. To assess the adequacy of the formulas for dynamic problems, a vibration test was conducted with the panel shown in Fig. 8 to recover the first 6 flexible modes. Because data are available, this panel is chosen as our second test-case. For completeness, two models have been developed; (1) a FEM using equivalent panel properties, hereafter referred to as the PAnel-Equivalent Model (PaEM), and (2) a FEM with the detailed panel topology, hereafter referred to as PAnel-Detailed Model (PaDM).

Modeling details for both FEMs, developed using MSC Nastran, are discussed next.

Although the panel geometry is relatively simple, the panel itself had a slight curvature, which made the modeling effort somewhat more difficult. The PaEM has 496 nodes (2976 DOF) and 450 CQUAD4 elements. Panel layers (i.e., skin and ribs) are modeled using composite laminate theory with isotropic properties for the skin and orthotropic properties for the rib; with properties computed using the isogrid design formulas [18]. To contrast, the PaDM model has 844 nodes (5064 DOF) and 810 CQUAD4 elements. Because the panel geometry is modeled explicitly in $\mathrm{PaDM}$, there is no need to approximate the cross-section properties. Table 2 list the first 6 modal frequencies with the panel in a near free-free condition. ELA is now applied to an analytically perturbed PaEM.

\subsection{Discussion of results for ELA with the PaEM model}

To exercise ELA, the PaEM FEM has been perturbed by adding an edge along the perimeter of the panel (not shown 
Frequency $(\mathrm{Hz})$

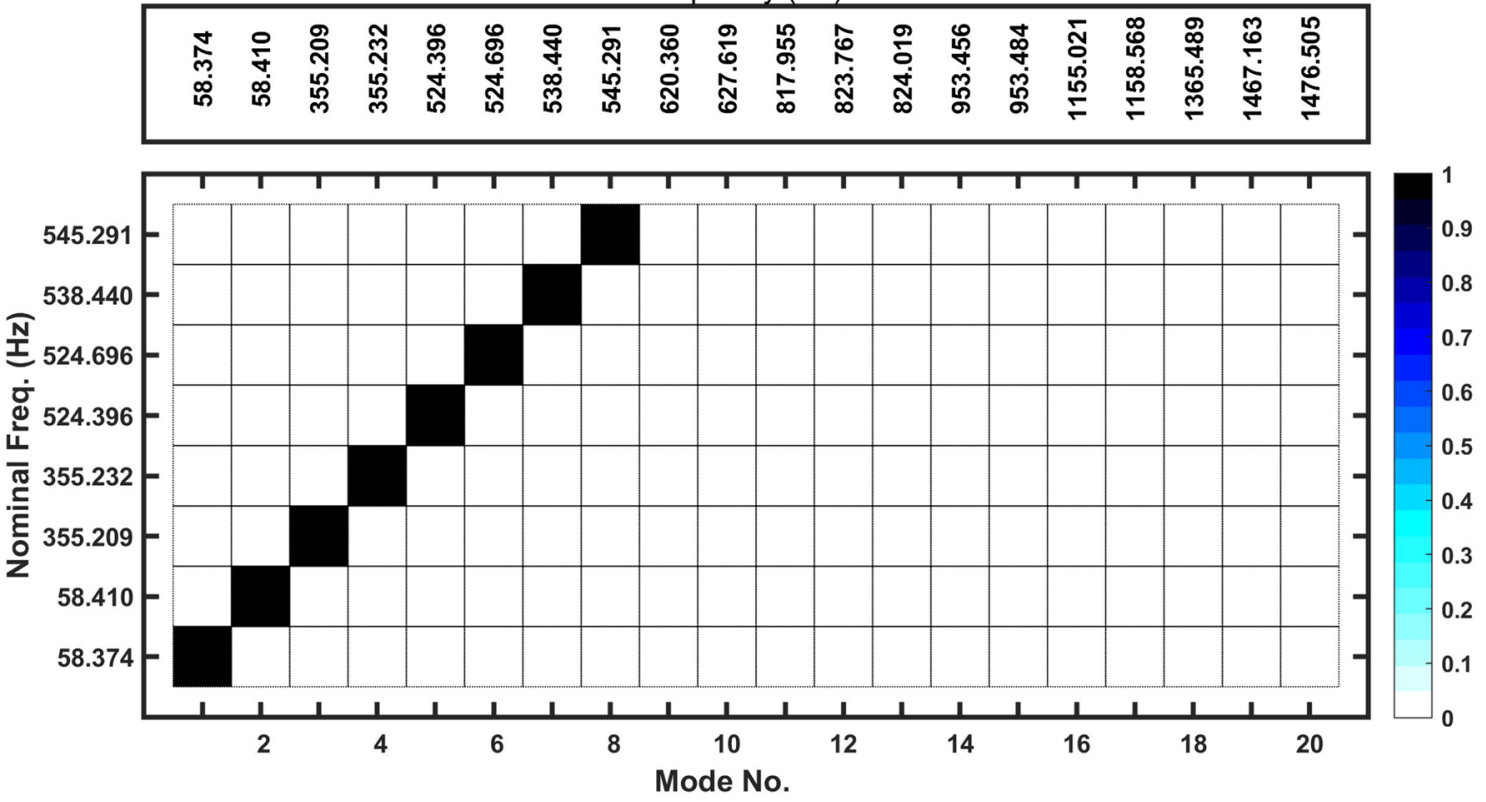

Fig. 4 Rectangular self-orthogonality matrix with 99 sensors

Fig. 5 Tube perturbed area

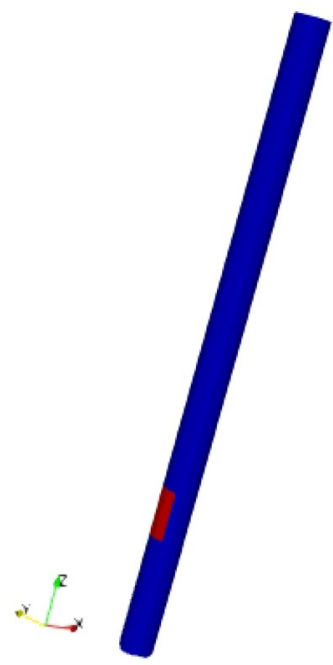

because when plotted it is difficult to see). Of course the object of the exercise is for ELA to point out areas of the model that need to be corrected based on measured data. In lieu of measured data, six modes from the perturbed model are used. To be consistent with standard modal test practices, shape data at only 23 locations, selected from an optimal sensor placement study, are used to ensure that SO values for off-diagonal terms are less than $10 \%$. Figure 9 shows the spatial distribution of the 23 locations where shape information is evaluated. In addition, Table 2 shows the frequencies for PaEM with the added edge, referred to as PaEM-WE, for comparison to the original model. Figure 10 shows results from applying ELA using 10 modes and 23 locations. The top plot of Fig. 10 are results using CoMAC, middle plot are results for $\mathrm{RME}$, and bottom plot are results for ADMI. As before, numerical values with ELA are sorted into three areas of descending magnitudes. Red colors correspond to the highest ELA index value, or areas in need of the largest corrections. Because the model is perturbed along the edges, ELA results should flag those areas. Only the ADMI algorithm shows the highest error areas along the perimeter. In addition, ADMI calls for stiffness increases for all flagged areas and negligible mass corrections. RME also flagged some areas along the two extreme edges, whereas CoMAC missed the problem areas completely.

Based on results from these two ELA application examples, the ADMI algorithm not only identifies areas of improvements correctly but also the need to increase or decrease values. Although RME has shown some success in identifying problem areas, it does not provide the type of changes needed to correct the model. For this reason, only ADMI is used next with panel test data to calibrate the PaEM FEM.

\section{Isogrid panel modal test}

A discussion of the isogrid panel modal test is presented first along with comparisons to the FEM. To minimize the impact of boundary conditions during the test, the panel is tested in a vertical orientation while simply supported using rubber-bands at four locations, as shown in Fig. 11; 
Frequency $(\mathrm{Hz})$

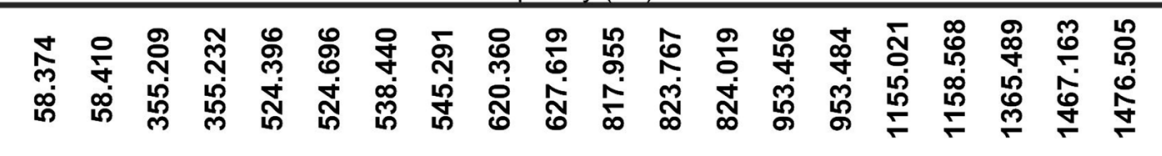

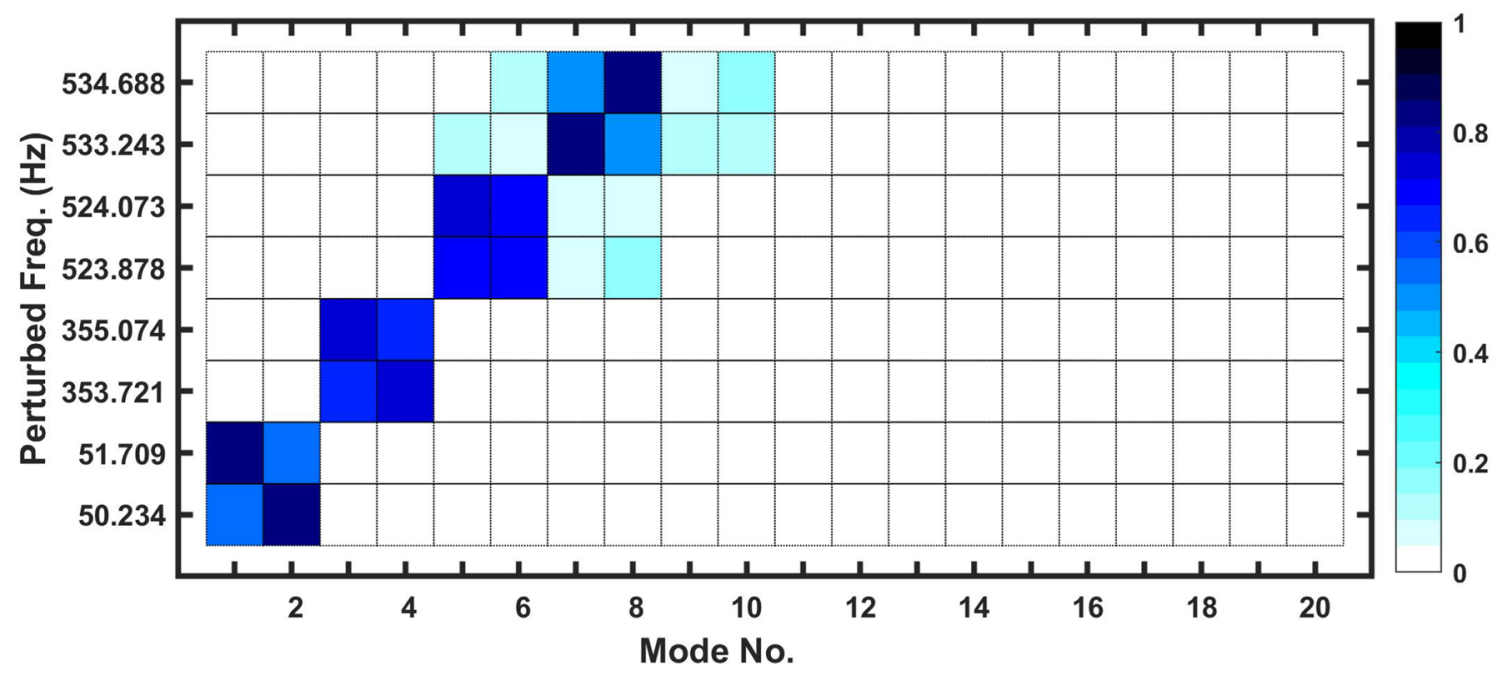

Fig. 6 Cross-orthogonality for the perturbed and original model

Fig. 7 ELA results using CoMAC, RME, and ADMI with 10 modes and 99 sensors

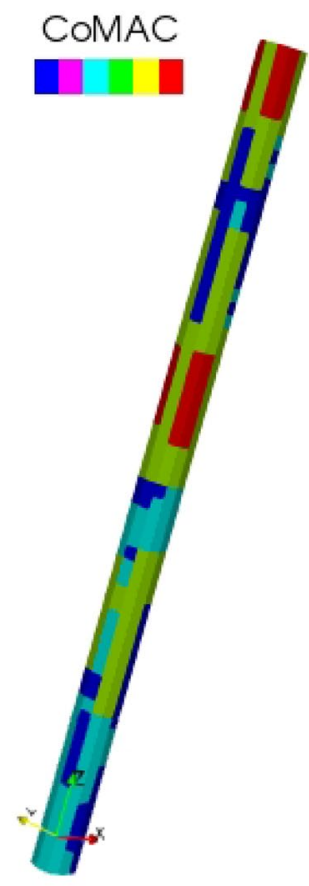

two on top and two at the bottom (gravity vector from top to the bottom of the figure). A Polytec Laser Vibrometer [19] system is used to collect Frequency Response Functions (FRF) from impacts with a calibrated impact hammer. FRFs are computed from 10 averages at each of the 23 locations with frequency resolution $\Delta F=5.12 \mathrm{~Hz}$.
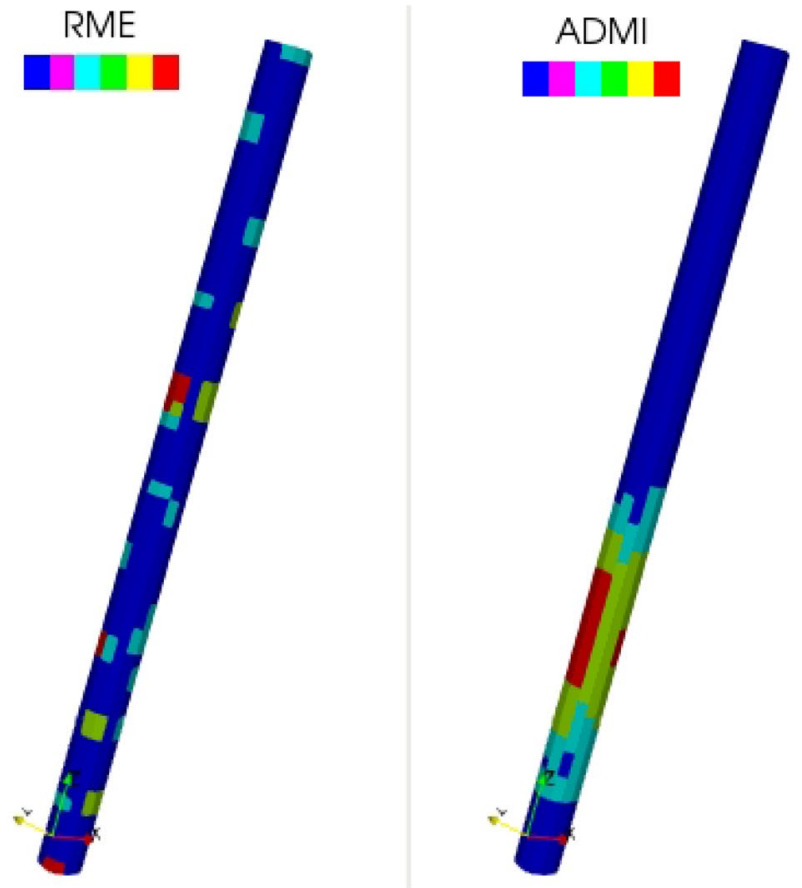

Figure 12 shows the principal value FRF using all 23 locations; test data are shown with a solid-line and the dashed-line is the identified model using the Eigensystem Realization Algorithm (ERA) [20]. Frequencies from the identification process and both FEMs are shown in Table 3. Excluded from Table 3 are six pendulum modes, all under 


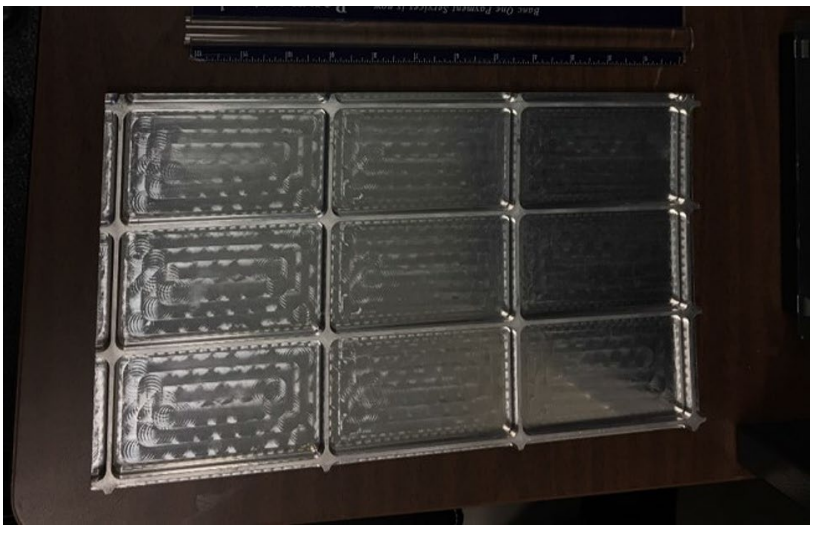

Fig. 8 Rectangular isogrid panel

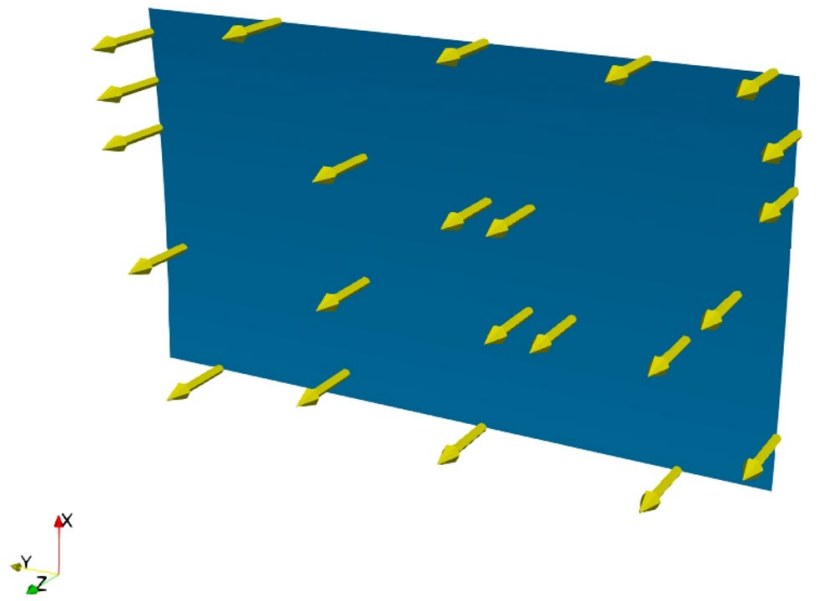

Fig. 9 Panel sensor placement with 23 locations

Table 2 First 6 free-free modes of isogrid panel

\begin{tabular}{llll}
\hline Mode No. & PaDM Freq. $(\mathrm{Hz})$ & PaEM Freq. $(\mathrm{Hz})$ & $\begin{array}{l}\text { PaEM-WE } \\
\text { Freq. }(\mathrm{Hz})\end{array}$ \\
\hline 1 & 115.4 & 107.7 & 114.5 \\
2 & 385.8 & 359.2 & 378.2 \\
3 & 457.4 & 392.5 & 437.8 \\
4 & 696.4 & 767.8 & 791.9 \\
5 & 813.3 & 795.7 & 881.2 \\
6 & 918.0 & 948.5 & 992.0 \\
\hline
\end{tabular}

$35 \mathrm{~Hz}$. Results from the identification process, labeled ERA$I D$, show that the PaDM maximum frequency error is 2.4 $\%$, whereas for PaEM the maximum frequency error is as much as $12.2 \%$. To compare mode shapes, Fig. 13 shows $\mathrm{CO}$ results using PaEM and Fig. 14 shows $\mathrm{CO}$ results using PaDM. Color bars in increments of $5 \%$ are used to show the orthogonality values. $\mathrm{CO}$ off-diagonal values with both

SN Applied Sciences
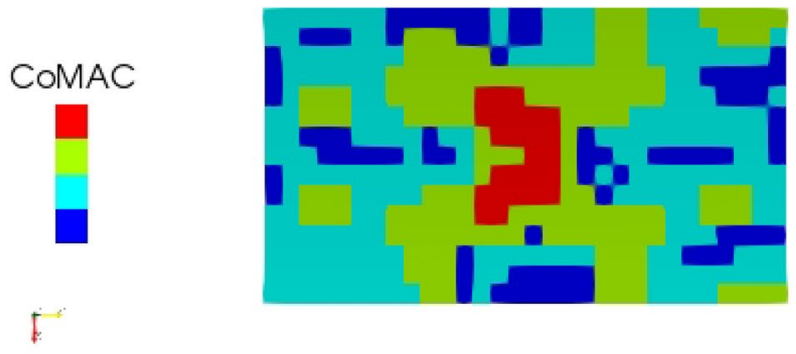

RME
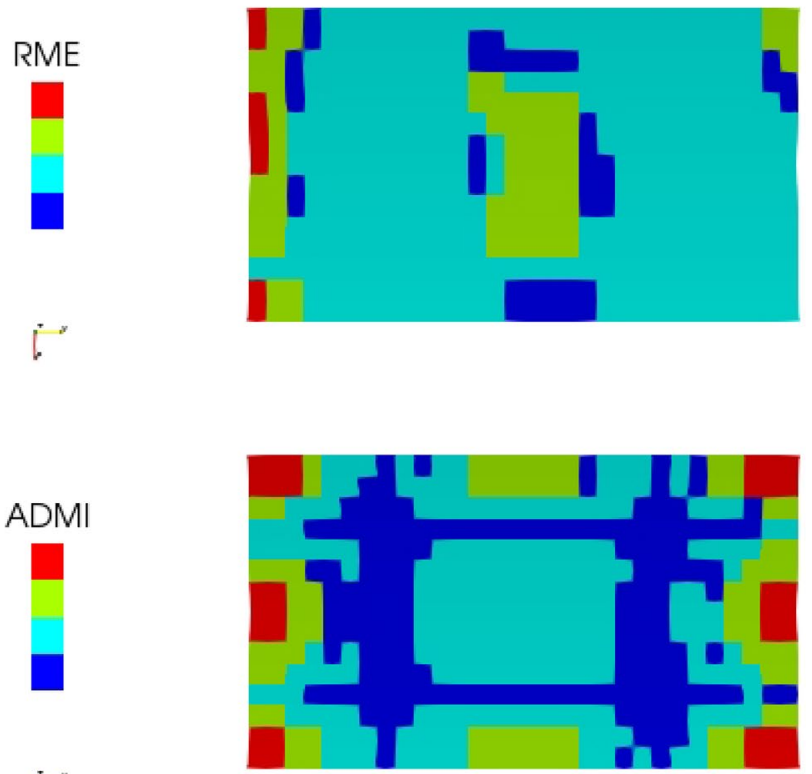

Fig. 10 ELA results using simulated data CoMAC (Top), RME (middle), and ADMI bottom

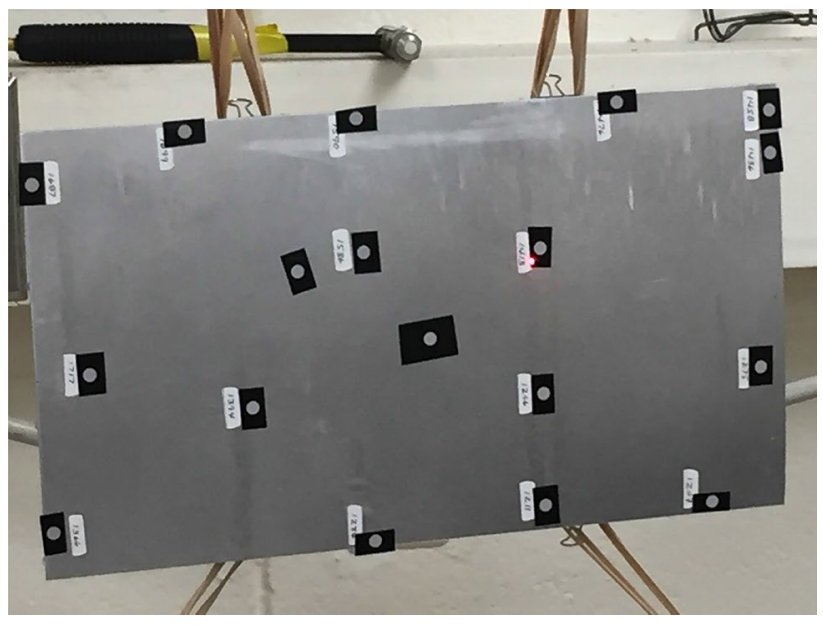

Fig. 11 Panel test configuration 


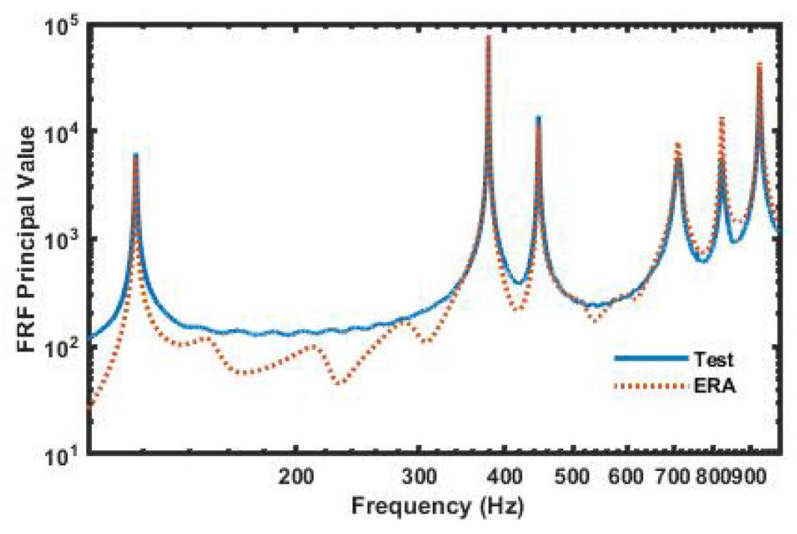

Fig. 12 FRF PV from 23 sensor locations modeling approaches are about the same, but PaDM predicts mode number 6 , in the proper order. Because PaDM frequencies are so close to the test, only PaEM is used with ELA for model calibration.

\subsection{Discussion of results using modal test data}

To illustrate the ELA process with test data, the PaEM is used to identify model areas in need of corrections. For completeness, results for all three ELA approaches with the test data are shown on Fig. 15; top results are for CoMAC, middle results are for $\mathrm{RME}$, and bottom results are for ADMI. As before, red colors correspond to areas where the largest changes are needed. Only results from the ADMI are used in the following calibration exercise. Because

Table 3 Comparison of test with model frequencies

\begin{tabular}{lllllrrr}
\hline Mode No. & ERA-ID Freq. (Hz) & PaDM Freq. (Hz) & $\begin{array}{l}\text { PaDM Freq. } \\
\text { Error (\%) }\end{array}$ & PaEM Freq. (Hz) & $\begin{array}{l}\text { PaEM Freq. } \\
\text { Error (\%) }\end{array}$ & $\begin{array}{l}\text { PaEM-Cal } \\
\text { Freq. (Hz) }\end{array}$ & $\begin{array}{l}\text { PaEM-Cal } \\
\text { Freq. Error } \\
(\%)\end{array}$ \\
\hline 1 & 117.0 & 115.4 & -1.4 & 107.7 & -7.9 & 120.0 & 2.6 \\
2 & 378.3 & 385.8 & 2.0 & 359.2 & -5.0 & 349.5 & -7.6 \\
3 & 446.8 & 457.4 & 2.4 & 392.5 & -12.2 & 413.2 & -7.5 \\
4 & 709.6 & 696.4 & -1.9 & 767.8 & 8.2 & 763.6 & 7.6 \\
5 & 821.5 & 813.3 & -1.0 & 795.7 & -3.1 & 831.5 & 1.2 \\
6 & 930.8 & 918.0 & -1.4 & 948.5 & 1.9 & 956.7 \\
\hline
\end{tabular}

Frequency $(\mathrm{Hz})$

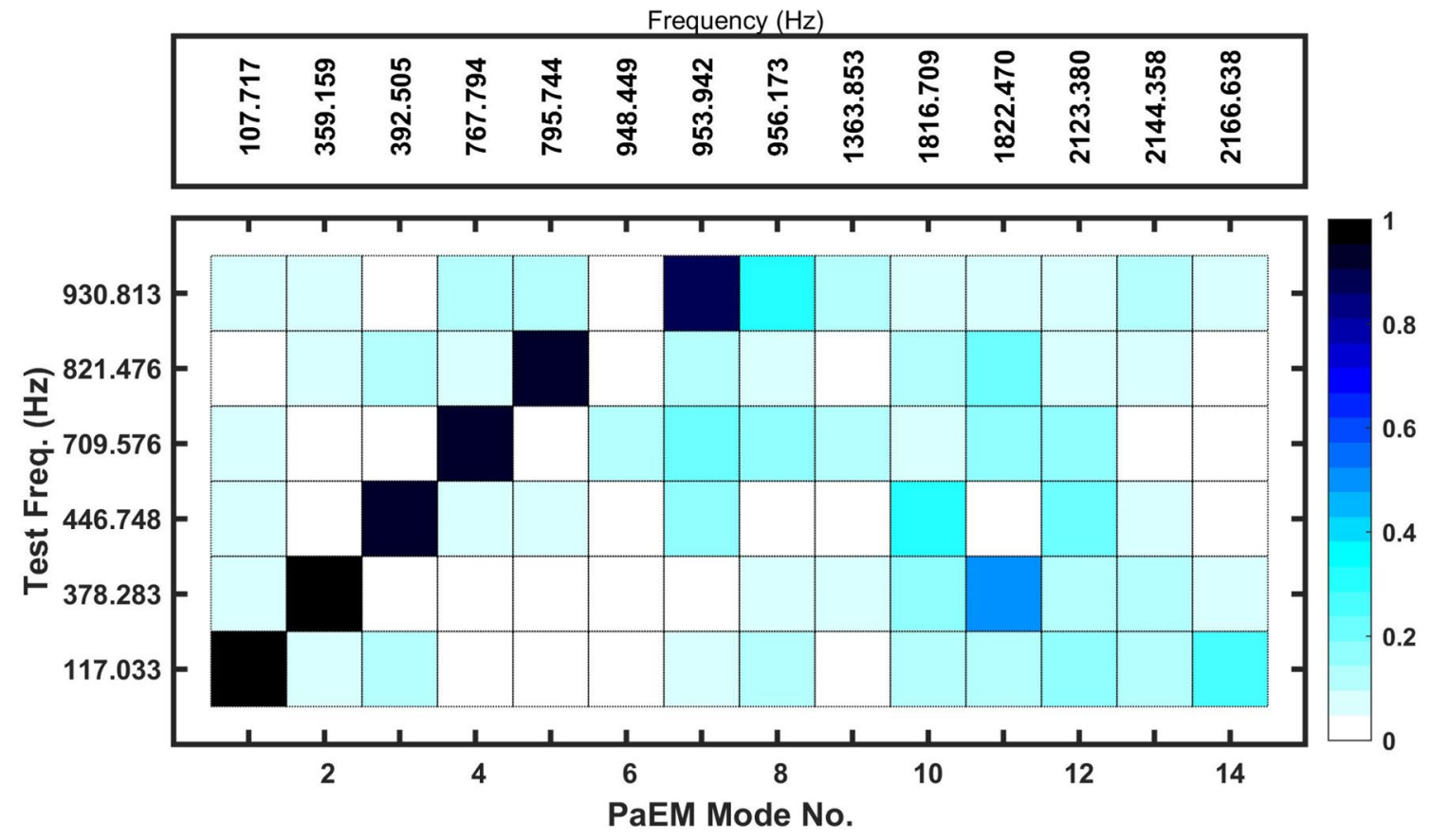

Fig. 13 Cross-orthogonality of identified model versus PaEM 
Frequency $(\mathrm{Hz})$

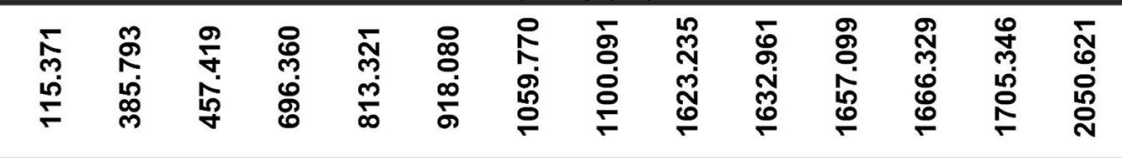

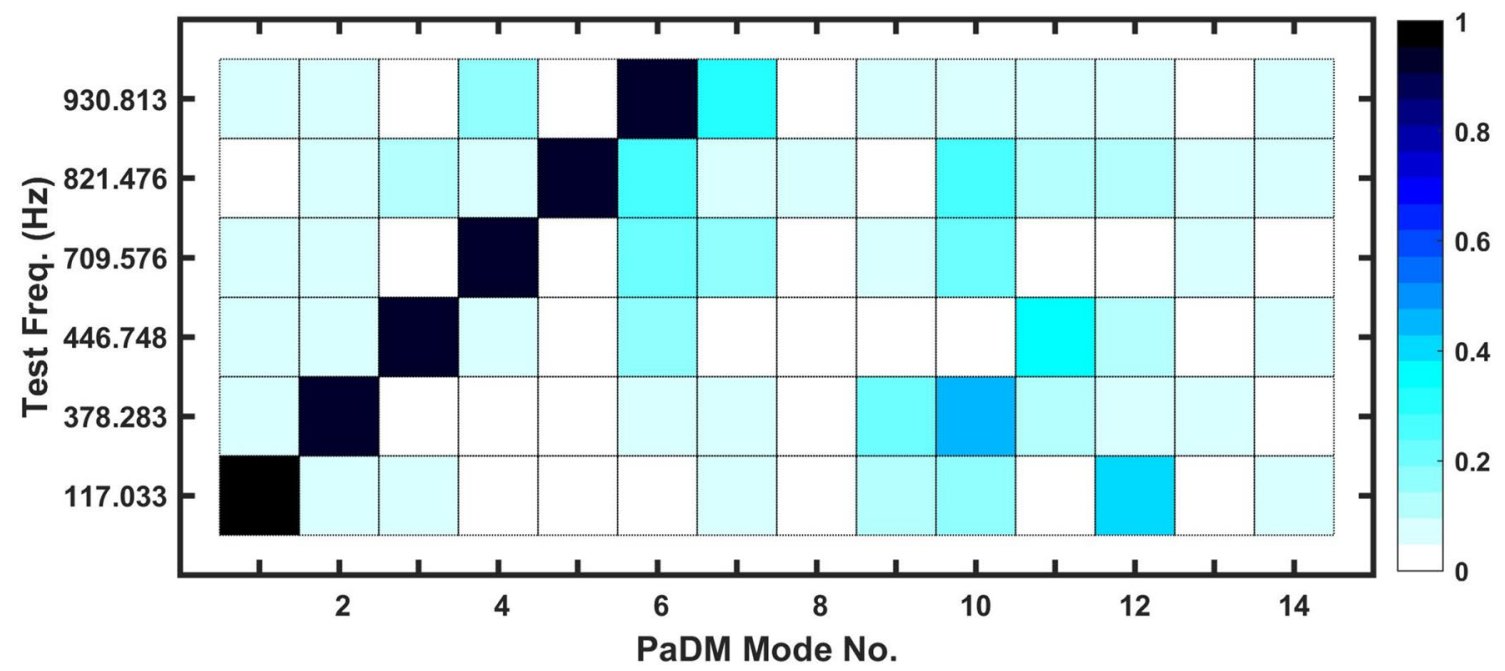

Fig. 14 Cross-orthogonality of identified model versus PaDM

changes in mass and stiffness, as computed using ADMI, are unconstrained corrections to the nominal model (i.e., non-existing connections are possible), the ADMI changes are not necessarily linked to physical parameters in the FEM. Therefore, our ability to compute an ADMI solution does not guarantees our ability to find matching parameters for the FEM. In this example, the ADMI algorithm identified the need to increase both mass and stiffness in those areas shown in Fig. 15. Because mass and stiffness increases vary throughout the different zones, corrections here are implemented by changing the thickness of the material. To simplify the implementation, the ADMI recommended changes are partitioned over 5 zones using 10 thickness parameters. Zones are partitioned based on magnitude of the needed changes; (largest change corresponds to zone 1). Certainly, users have the option to select an arbitrary number of zones (or magnitude thresholds) to implement the changes, which allows for an arbitrary number of parameters. Model calibration is discussed next.

Sensitivity results for this problem are not shown, but results confirmed that all 10 parameters had very similar importance up to $1000 \mathrm{~Hz}$. Therefore, no reduction in the number of parameters is justified.

\section{Model calibration of isogrid panel}

Starting with the ELA corrections, model changes are partitioned into 5 zones; the next step is a search for FEM parameters to reconcile test with the analysis (i.e., model calibration). Although there are several model calibration tools commercially available, to our knowledge, ELA is not implemented in any of the existing tools. For convenience, our implementation here follows closely the same approach used in [8]. In the future, commercial codes should implement ELA to facilitate the identification and partitioning of the model into repair zones. For now, this process is accomplished by manipulating the FEM input file using MATLAB [21] programming language.

An important aspect of the calibration process is the definition of appropriate metrics to gage the adequacy of the model. Often, aerospace manufacturers must ensure closeness of model frequencies and mode shapes to meet load and stability margins. Towards that goal, define the maximum frequency error as a function of the parameter vector $p$ as $E_{f}(p)$, and the maximum $C O$ off-diagonal value as $E_{o}(p) . E_{f}(p)$ is the maximum difference between a paired set of modal frequencies between test and analysis, whereas $E_{o}(p)$ is a scalar with the maximum off-diagonal value in the $\mathrm{CO}$ matrix, computed using mass-normalized modes. A way to incorporate both errors into a scalar value for optimization is to define 


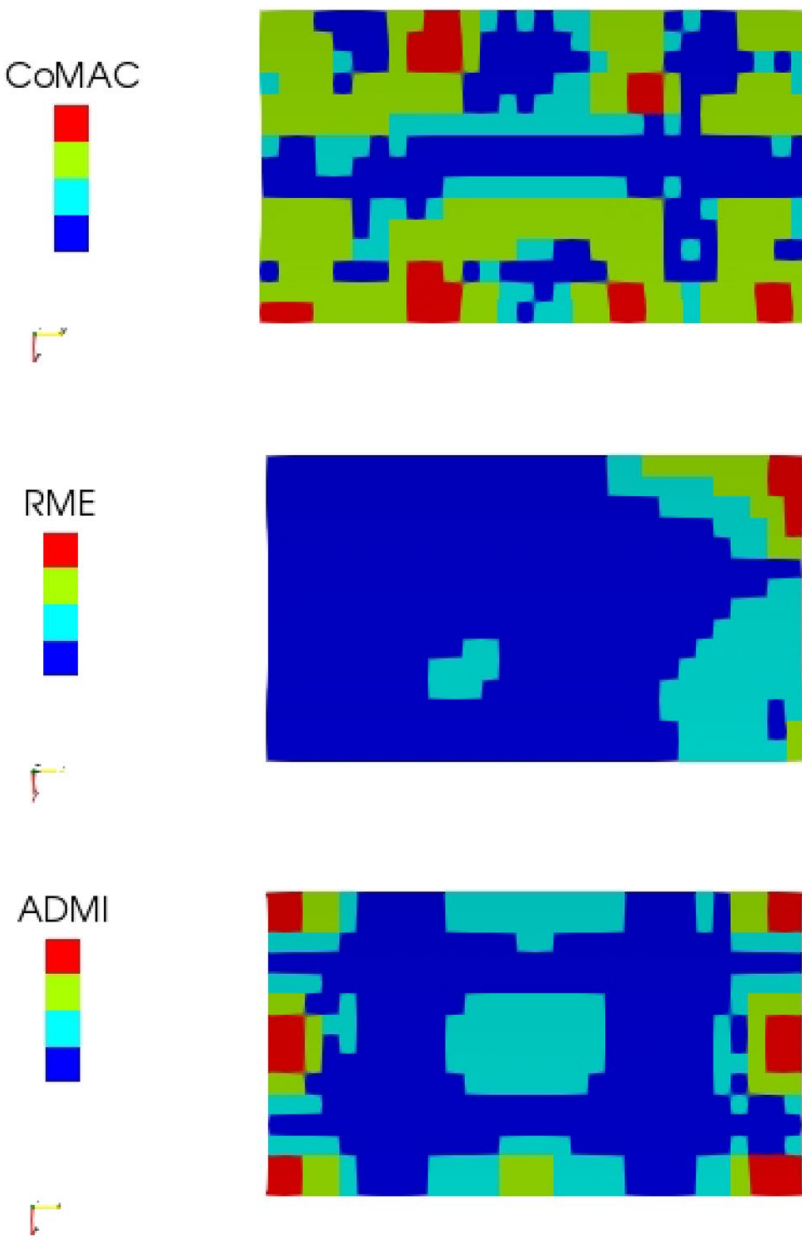

Fig. 15 ELA results using 6 measured modes; Top-CoMAC, MiddleRME, and Bottom-ADMI

$J(p)=\left\|\begin{array}{l}w_{1} E_{f}(p) \\ w_{2} E_{o}(p)\end{array}\right\|_{2}$

which uses the 2-norm to combine these two metrics. Weighting factors $w_{1}$ and $w_{2}$ are used to properly scale both frequency and $\mathrm{CO}$ values. Note that before computing the maximum frequency error, one must pair test frequencies with analysis using orthogonality. This step uses a pre-set threshold of orthogonality to decide what measured modes correspond to analysis modes; in our problem 0.8 is used. Finally, during optimization our objective is to minimize $J(p)$ using $w_{1}=1.0$ and $w_{2}=0.1$.

Before attempting an optimization solution, it is important to assess the probability that a solution exists within the parameter values and ranges selected. Oftentimes, models are simply not able to explain test observations and therefore, to seek an optimization solution is pointless. To address this problem, pre-optimization analysis is performed first, where parameter values are assumed to
Table 4 Parameter definitions for PaEM with 5 zones

\begin{tabular}{llllll}
\hline Zone No. & $\begin{array}{l}\text { Descrip- } \\
\text { tion }\end{array}$ & $\begin{array}{l}\text { Thickness } \\
\text { (in) }\end{array}$ & $\begin{array}{l}\text { Upper } \\
\text { limit fac- } \\
\text { tor }\end{array}$ & $\begin{array}{l}\text { Lower } \\
\text { limit fac- } \\
\text { tor }\end{array}$ & $\begin{array}{l}\text { Cal. } \\
\text { Solution }\end{array}$ \\
\hline 1 & Rib & 0.342 & 2.0 & 1.0 & 1.000 \\
1 & Skin & 0.098 & 2.0 & 1.0 & 1.000 \\
2 & Rib & 0.342 & 1.8 & 1.0 & 1.000 \\
2 & Skin & 0.098 & 1.8 & 1.0 & 1.336 \\
3 & Rib & 0.342 & 1.5 & 1.0 & 1.140 \\
3 & Skin & 0.098 & 1.5 & 1.0 & 1.500 \\
4 & Rib & 0.342 & 1.2 & 1.0 & 1.000 \\
4 & Skin & 0.098 & 1.2 & 1.0 & 1.000 \\
5 & Rib & 0.342 & 1.1 & 1.0 & 1.003 \\
5 & Skin & 0.098 & 1.1 & 1.0 & 1.002 \\
\hline
\end{tabular}

be uniformly distributed over user-defined ranges. This pre-optimization step executes the FEM multiple times to create a family of solutions, to answer two fundamental questions: (1) how probable is it that the FEM can explain test observations, and (2) what parameters are the most important. Probability is estimated from the sampled set of solutions, whereas parameter importance is established using correlation analysis. Although, parameter importance can be easily computed using conventional sensitivity, correlation analysis is used instead. Certainly, the pre-optimization step is an additional computation burden, but in the end, it helps tremendously to decide if the current FEM and parameter values are appropriate to meet test observations.

\subsection{Discusion of model calibration results}

To begin the pre-optimization screening process, the PaEM bulk data file is modified to have separate thicknesses associated with each of the 5 zones, as listed in Table 4 . Each zone has a skin and rib upper and lower limit factors that are used as multipliers to create a sampled set of parameter values. A family of FEM solutions is computed using 69 parameter sets created using MATLAB D-optimal sampling. Instead of viewing responses at each individual sensor, the ordinate in Fig. 16 shows the Principal Value (PV) as a function of frequency, computed using 23 sensors, test is solid-blue and the analysis PV envelope in dashed-red is created from all 69 FEM solutions. With only one impact location, the principal value at a particular frequency is the maximum singular value of the FRF matrix. If the model is to explain the test, test results must fall within the analysis PV envelope. If not, the probability that the FEM will predict responses outside the envelope is less than $1 / 69$. The only exception is at a resonant frequency 
Fig. 16 Principal value envelope for analysis (dashed-red) versus test (solid-blue)

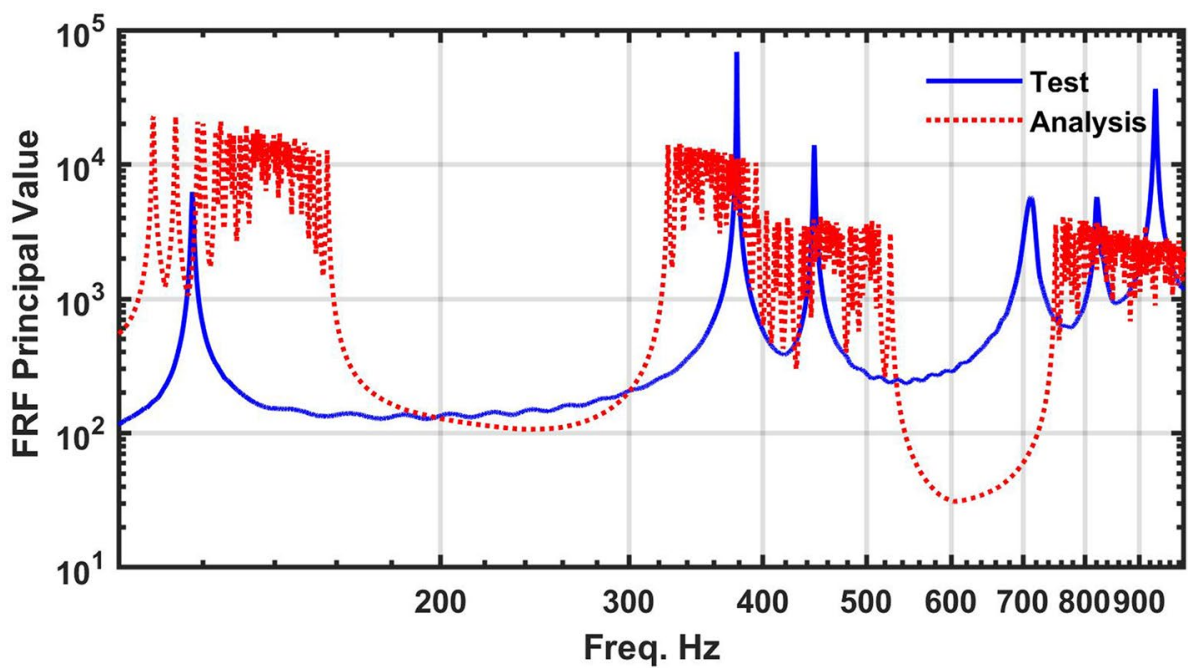

because the FEM modal damping value of $1 \%$ is arbitrarily over-prescribed. With these parameters and the current FEM, the probability that the model will predict a panel frequency at $709.6 \mathrm{~Hz}$ is less than $1 / 69$. Knowing this, if users decide to proceed with optimization, at least their expectations are in line with the model capability. Although not apparent, the determination of parameter limits in Table 4 is a time consuming trial-and-error process that is critical to the success of the optimization process.

For illustration purposes, an optimization solution, using a Genetic algorithm, is computed to reconcile test with the PaEM. The optimized parameter set, found after an exhaustive search, is shown in Table 4 under "Cal-Solution"; the corresponding PaEM-Cal frequencies are shown in Table 3. CO results, using the optimized parameter set, are shown on Fig. 17; ordinate is test and abscissa shows the model frequencies using parameters in the "Cal-Solution". Note that the "Cal-solution" increased one parameter in zone 3 by $50 \%$ while other parameters are at their lowest limit (often an indication of an over-constrained problem). On the other hand, $E_{o}(p)$ values have improved, when compared to the nominal model in Fig. 13, and now the measured mode number 6 is aligned with the model. No single modal

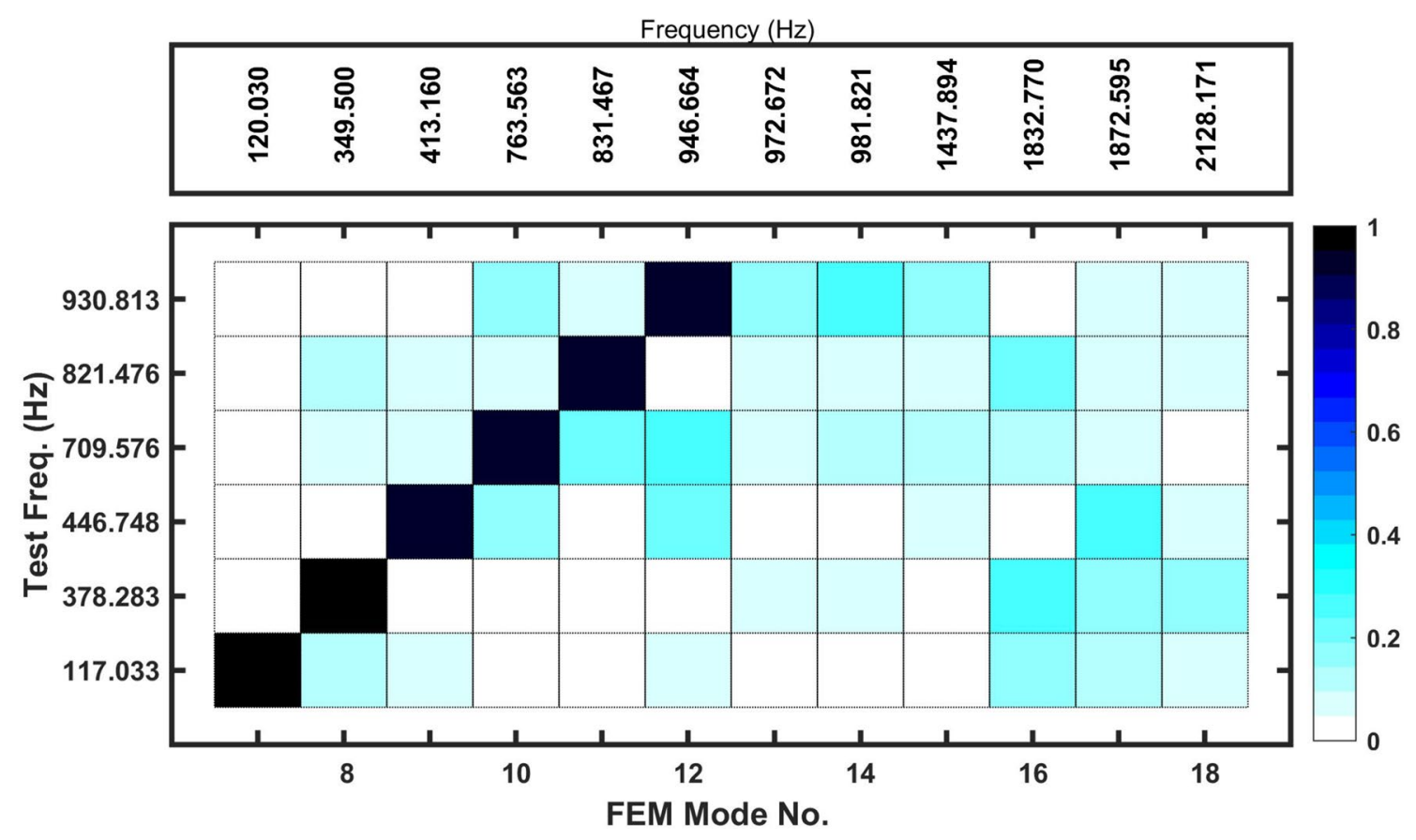

Fig. 17 Cross-orthogonality results with the calibrated model 
frequency is matched exactly, but users can enforce frequency matching of at least one mode by simply changing the weights in the objective function. Using the parameters in "Cal-Solution", the optimizer decreased the overall frequency error from 12.2 to $7.6 \%$, as shown in Table 3. Also, with thickness changes, the total system mass changed from 1.35 to $1.41 \mathrm{lbf}$, which is a $4.4 \%$ increase. At this point, additional model improvements without fundamental model changes are not likely.

Adequacy of calibration efforts are often gaged on the ability of the process to reconcile the model with test. However, our inability to fully reconcile the PaEM with test is not due to the calibration process. Instead, the problem is an inadequate representation of the geometrical characteristics of the panel implemented in the PaEM. To contrast, when comparing the test results to the PaDM, frequencies are within $2.4 \%$ from the start. Readers are reminded that the PaEM contains an equivalent representation of the geometry, whereas, PaDM contains the correct geometrical features. Consequently, fundamental model flaws, like geometrical discrepancies, must be corrected before calibration efforts are undertaken.

\section{Conclusions}

Three Error Localization Algorithms (ELA) namely the: (1) Coordinate Modal Assurance Criterion (Co-MAC), 2) Robust Model Error (RME), and (3) Analytical Dynamic Model Improvement (ADMI) are evaluated to identify model flaws using both simulated and measured mode shapes and frequencies. All three approaches rely on a nominal Finite Element Model (FEM) to determine model improvement areas. To ascertain the adequacy of the ELA approaches, modes and frequencies from two example problems with known perturbed regions are used to compare ELA approaches. Only RME and ADMI are able to identify known problem areas. However, only the ADMI algorithm computes the necessary changes to the nominal model to match the test data exactly. These changes provide users not only with problem areas, but also, with correction magnitudes for both mass and stiffness.

In order to implement ELA with practical problems, users must ensure that the test data contains the proper information about the modes of interest. Careful pretest planning is critical when selecting number of sensors, optimal sensor placement, and modes to meet test metrics. Effectively, this ensures that modes and frequencies of interest are distinguishable from one another. Just as important is the development and selection of the nominal model and candidate set of degrees-of-freedom (DOF). When the candidate set is coarse, the ability to identify problem areas is practically impossible.
Finally, the paper presents results from an application of the ADMI algorithm to vibration test data of an isogrid panel. ADMI is used to identify problem areas first, followed by an implementation of model calibration. Results from ELA indicates that both stiffness and mass changes are needed to reconcile the model. Changes are implemented by assigning and adjusting independently the thickness of the skin and ribs for each of the five identified zones. Although the model can be significantly improved with calibration, the fact is that fundamental flaws oftentimes are not correctable without the need for additional modeling efforts. In the example shown, a FEM using the detailed geometry of the isogrid panel produced frequency results closer to test than the calibrated model. For this reason, model calibration should only be attempted after a comprehensive modeling effort is completed.

\section{Compliance with ethical standards}

Conflict of interest On behalf of all authors, the corresponding author states that there is no conflict of interest.

\section{References}

1. Friswell MI, Mottershead JE (1996) Finite element model updating in structural dynamics. Copyright Kluwer Academic Publisher, Reprinted. ISBN 0-7923-3431-0 (1995)

2. Maia NMM, Silva JMM, He J, Lieven NAJ, Lin RM, Skingle GW, To W-M, Urgueira APV (1997) Theoretical and experimental modal analysis. Research Studies Press LTD, ISBN 0-471-97067-0

3. Anonymous (1998) Guide for the verification and validation of computational fluid dynamics simulations. AIAA, G-077-1998

4. Anonymous (2006) Guide for verification and validation in computational solid mechanics. ASME, Verification and Validation 10-2006

5. Ewins DJ (2000) Modal testing theory, practice, and application, 2nd edn. Copyright Research Studies Press Ltd

6. Blelloch PA, Vold H (2005) Orthogonality of large modelsWhat's the problem. In: Proceedings of the 23rd international modal analysis conference (IMAC)

7. Blelloch PA (2006) Cross-orthogonality of closely spaced modes. In: Proceedings of the 24th international modal analysis conference (IMAC)

8. Horta LG, Reaves MC, Buehrle RD, Templeton JD, Lazor DR, Gaspar JL, Parks RA, Bartolotta PA (2011) Finite element model calibration for ares I-X flight vehicle. J Eng Mech. https://doi. org/10.1001/s11340-010-9456-X

9. Allemang RJ, Brown DL (1982) A correlation coefficient for modal vector analysis. In: Proceedings of the international modal analysis conference (IMAC), pp 110-116

10. Allemang RJ (2003) The modal assurance criterion (MAC): twenty years of use and abuse. J Sound Vib 37(8):14-23

11. Alvin KF (1995) Robust model error localization for damage detection and finite element model update. In: Proceedings of 6 th international conference on adaptive structures, PA, pp 305-315 
12. Farhat C, Hemez FM (1993) Updating finite element dynamic models using an element-by-element sensitivity methodology. AIAA J 31(9):1702-1711

13. Wei F-S (1990) Analytical dynamic model improvement using vibration test data. AIAA J 28:175-177

14. Anonymous (2019) MSC Nastran multidisciplinary structural analysis. The MacNeal-Schwendler Corporation, https://www. msc.software.com

15. Guyan RJ (1965) Reduction of stiffness and mass matrices. AIAA J 3(2):380

16. O'Callahan JC, Avitabile P, Riemer R (1989) System equivalent reduction expansion process (SEREP). In: Proceedings of the 7th international modal analysis conference (IMAC)

17. Chu D, Chu M, Lin W-W (2009) Quadratic model updating with symmetry, positive definiteness, and no-spill-over. SIAM J Matrix Anal Appl 31(2):546-564
18. Anonymous (1973) Isogrid design handbook. Prepared by McDonnel Douglas Astronautics Company, MDC-G4295A, Contract NAS 8-28619, Revised April 2004

19. Anonymous (2009) Polytec scanning vibrometer software manual 8.7. Polytec Inc., North American Headquarters, Tustin, CA, http://www.polytec.com

20. Horta LG, Juang J-N (1993) Frequency domain system identification methods: matrix fraction description approach. In: Proceedings of the 1993 guidance, navigation, and control conference, paper No. 93-3839

21. Anonymous (2018) MATLAB R-2018B, Copyright 1994-2019, The Mathworks Inc. http://www.mathworks.com

Publisher's Note Springer Nature remains neutral with regard to jurisdictional claims in published maps and institutional affiliations. 